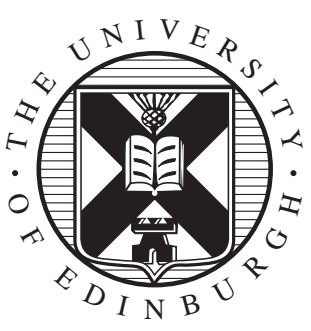

Division of Informatics, University of Edinburgh

Centre for Intelligent Systems and their Applications

From Approximative to Descriptive Fuzzy Classifiers

by

Javier Gomez-Marin-Blazquez, Qiang Shen

Informatics Research Report EDI-INF-RR-0126 


\title{
From Approximative to Descriptive Fuzzy Classifiers
}

\author{
Javier Gomez-Marin-Blazquez, Qiang Shen \\ Informatics Research Report EDI-INF-RR-0126 \\ DIVISION of INFORMATICS \\ Centre for Intelligent Systems and their Applications
}

May 2002

Presented in: IEEE Transactions on Fuzzy Systems

\begin{abstract}
:
This paper presents an effective and efficient approach for translating fuzzy classification rules that use approximative sets to rules that use descriptive sets and linguistic hedges of predefined meaning. It works by first generating rules that use approximative sets from training data and then translating the resulting approximative rules into descriptive ones. Hedges that are useful for supporting such translations are provided. The translated rules are functionally equivalent to the original approximative ones, or a close equivalent given search time restrictions, while reflecting their underlying preconceived meaning. Thus, fuzzy descriptive classifiers can be obtained by taking advantage of any existing approach to approximative modeling which is generally efficient and accurate, whilst employing rules that are comprehensible to human users. Experimental results are provided and comparisons to alternative approaches given.
\end{abstract}

Keywords : Fuzzy classification, descriptive modelling, approximative modelling, linguistic hedges

Copyright (C) 2002 by The University of Edinburgh. All Rights Reserved

The authors and the University of Edinburgh retain the right to reproduce and publish this paper for non-commercial purposes.

Permission is granted for this report to be reproduced by others for non-commercial purposes as long as this copyright notice is reprinted in full in any reproduction. Applications to make other use of the material should be addressed in the first instance to Copyright Permissions, Division of Informatics, The University of Edinburgh, 80 South Bridge, Edinburgh EH1 1HN, Scotland. 


\title{
From Approximative to Descriptive Fuzzy Classifiers
}

\author{
Javier G. Marín-Blázquez and Qiang Shen \\ \{javierg, qiangs\}@dai.ed.ac.uk \\ Division of Informatics \\ The University of Edinburgh, UK
}

\begin{abstract}
.
This paper presents an effective and efficient approach for translating fuzzy classification rules that use approximative sets to rules that use descriptive sets and linguistic hedges of predefined meaning. It works by first generating rules that use approximative sets from training data and then translating the resulting approximative rules into descriptive ones. Hedges that are useful for supporting such translations are provided. The translated rules are functionally equivalent to the original approximative ones, or a close equivalent given search time restrictions, while reflecting their underlying preconceived meaning. Thus, fuzzy descriptive classifiers can be obtained by taking advantage of any existing approach to approximative modeling which is generally efficient and accurate, whilst employing rules that are comprehensible to human users. Experimental results are provided and comparisons to alternative approaches given.
\end{abstract}

\section{INTRODUCTION}

In applications such as systems monitoring and medical diagnosis, domain attributes often emerge from an elusive vagueness, a readjustment to context or an effect of human imprecision. The use of the soft boundaries of fuzzy sets, namely the graded memberships, allows subjective knowledge to be incorporated in describing these attributes and their relationships. Fuzzy techniques have proven to be very successful for creating, for example, robust controllers and user-friendly classifiers [1], [2], [3] to address such problems. Even when precise knowledge is available, fuzziness may be a concomitant of complexity involved in the reasoning process. Among the interesting features of fuzzy approaches is the potential of fuzzy production rules in attaching meaningful labels to the fuzzy sets [4], thereby allowing a human comprehensible representation of the system under consideration.

Fuzzy rule bases are typically assumed to be given by domain experts. However, the acquisition of knowledge on rule structures often forms the bottleneck to advance the success of fuzzy systems in practice [5], though the linguistic labels or fuzzy sets that are used within the rules may be subjectively defined. For many applications, there exists a considerable volume of historical data obtained by observing the behavior of the system concerned. It is therefore desirable to be able to automatically generate rules from given data. Many techniques exist for this, most of which follow the so-called approximative approach ${ }^{1}$, which works by creating and tuning the fuzzy rule bases to best fit the data. The rules generated are not encoded to keep the meaning of the linguistic labels of the fuzzy sets used. Such an approach is, under minor restrictions, functionally equivalent to neural networks [6] and the resulting systems offer little explanatory power over their inferences.

Opposing approximative modeling stands the descriptive approach, in which model transparency is as important as accuracy. Prescribed fuzzy sets are either not allowed to be modified or, at most, are permitted to have very slight modifications. The descriptive sets used, i.e. the fuzzy sets defined by humans with a preconceived linguistic label attached, induce a fuzzy grid in the product space of the domain variables. As little modification is permitted, the grid and the hyperboxes delimited by these sets are almost fixed. Often these hyperboxes may contain examples of different output states and, as they are fixed, there is no way to separate these outputs directly.

It is possible to implicitly modify fuzzy rule bases without disrupting the definition of the underlying fuzzy sets, by the use of linguistic hedges [4] which allow more freedom in manipulating the

\footnotetext{
${ }^{1}$ The word approximative is used here instead of approximate to mirror the word descriptive in descriptive modeling which is itself an approximate approach.
} 
hyperboxes. Not all pure descriptive methods ${ }^{2}$ support the addition of hedges though (e.g. the well established work as reported in [2]). When no hedges can be applied an increase in the number of fuzzy sets, the addition of confidence factors or prioritizing some data as critical, may increase the performance. However, these methods typically also give rise to a loss in the interpretability.

Approximative approaches avoid the problem of fixed hyperboxes by changing the definitions of the fuzzy sets and hence the hyperboxes themselves. This ruins the underlying prespecified meaning attached to the fuzzy labels which have a natural appeal to the commonsense understanding of the words used. This is particularly so when a free or weakly constrained modification of fuzzy sets is carried out, even for approaches such as the one reported in [7]. The current literature pays little attention to this side-effect of using an approximative model and focuses on the accuracy of derived models (some even regarding such models as descriptive or interpretable), or simply maintains a descriptive model and accepts high modeling errors.

Recent attempts have been made to regain some of the fuzzy systems' transparency [8], [9], [10], by reducing otherwise possibly many antecedent approximative sets into a manageable number that possess interesting properties [11]. However, the linguistic labeling is done, when possible, a posteriori; the labels are attached to fuzzy sets generated by an approximative method but not to those given by humans. As the fuzzy sets used are self-clustered from training data and then given an artificial name, they may not have an intuitive interpretation. Human users of the resulting fuzzy systems have to do with the "friendly" words produced by the computer rather than the other way around. Significant work has been proposed to obtain descriptive explanations of approximative models [12] where each approximative rule fired is translated from one approximative hyperbox to one closest descriptive hyperbox. This represents an important departure from pure approximative modeling approaches. However, it adds on additional runtime cost and the explanation generated may not be sufficiently accurate due to the one-to-one approximate translation.

This paper presents an alternative approach, based on the initial investigations as reported in [13], [14], for producing descriptive fuzzy systems with a two-step mechanism. The first is to use an approximative method to create accurate rules and the second to convert the resulting approximative rules to descriptive ones. The conversion is, in general, one-to-many, implemented by a heuristic method that derives potentially useful translations and then by performing a fine tuning of these translations via evolutionary computation. Both steps are computationally efficient. The resultant descriptive system is ready to be directly applied for inference; no approximative rules are needed in runtime. Note that the work described here is focused on classification tasks.

The overall conversion process proposed is guided by functional equivalence rather than by similarity between approximative fuzzy sets and predefined descriptive ones in the antecedent part of the rules. To ensure a highly accurate translation, novel linguistic hedges are defined. The ultimate objective is to obtain a whole descriptive ruleset and to use it to perform the inference direct, thereby providing not only human comprehensible models but also straightforward explanation of the reasoning based upon the resulting models.

The rest of the paper is organized as follows. Section II describes the background and gives further detailed reasons for the present work. Section III proposes a set of useful linguistic hedges, which differ from those conventionally employed in the literature. Section IV presents the translation process, which maps approximative rules onto descriptive ones. It covers two methods, one based on the use of heuristics and the other on a genetic algorithm [15]. The latter is designed to use the result of the former as its initial population generator for efficiency purposes. Section $\mathrm{V}$ reports on typical experimental results, demonstrating the potential of the present research. The paper is concluded in section VI, with further work pointed out.

${ }^{2}$ Pure descriptive methods are those that do not allow any redefinition of the fuzzy sets used. 


\section{BACKGROUND AND JUSTIFICATIONS}

The task of descriptive modelling is to find a finite set of descriptive rules capable of reproducing the input-output behavior of the system being considered. For classification problems, without losing generality, the system to be modeled is assumed to be a MISO (Multi-Input Single-Output) one. That is, a system of $M$ inputs and one output that can be described by a set of $K$ rules such as:

$$
R_{i} \text { : If } x_{1} \text { is } D_{i}^{1} \text { and } \ldots \text { and } x_{M} \text { is } D_{i}^{M} \text { then } y \text { is } \text { Class }_{h}
$$

where $R_{i}$ is the $i$ th rule $(1 \leq i \leq K), x_{j}$ is the $j$ th input variable $(1 \leq j \leq M), y$ is the output variable to be assigned to one of the possible output classes, and $D_{i}^{j}$ are descriptive fuzzy sets for these variables. $D_{i}^{j}$ can be either a single descriptive fuzzy set or a combination of one or two hedges and a descriptive fuzzy set. Note that more than two hedges per variable are allowed in theory. However, a joint use of more than two hedges often destroys the readability of the resulting descriptive rules and hence is not desirable to be employed in practice. Descriptive fuzzy sets are human defined and fixed throughout both the modeling and the inference processes.

This follows the general principle of supervised learning [16]. The only information about the behavior of the system under consideration is assumed to be a (usually large) set of input-output example pairs, where for each instantiation of the input variables an associated class is indicated:

$$
\Omega=\left\{\left(x_{t 1}, x_{t 2}, \ldots, x_{t M}, y_{t}\right)\right\}=\left\{\left(x_{t}^{M}, y_{t}\right), t=1, \ldots, N\right\}
$$

The ruleset to be induced is required to approximate the function $\varphi: X^{M} \rightarrow$ Class $Y$ (that theoretically underlies the system behavior) in the most consistently possible way with the given examples of input-output pairs. It is assumed that the collection of the data examples represents the system behavior in the product space $\left(X^{M} \times Y\right)$, where $X^{p}=\left(X_{1} \times X_{2} \times \ldots \times X_{M}\right), X_{1}, X_{2}, \ldots, X_{p}$ are the domains of discourse of the inputs and $Y$ is the domain of the output classes.

It is, in general, computationally prohibitive to perform exhaustive search in the space of all possible combinations of descriptive sets (with or without the use of linguistic hedges), in order to obtain descriptive rules. This is due to "curse of dimensionality" [17], [18]. The higher the number of variables available, and/or the higher the cardinality of the fuzzy partition for each variable, the higher (exponentially) the number of possible rules. This is generally true for approximative models as well. In fact, the size of all possible rules associated to the general product ruleset $R_{\text {Set }}$ of a given system, allowing up to two hedges per fuzzy set for example, may be represented by:

$$
\left|R_{S e t}\right|=H_{L_{1} 1} \times H_{L_{1} 2} \times L_{1} \times \ldots \times H_{L_{M} 1} \times H_{L_{M} 2} \times L_{M} \times S=h^{2 \times M} \times s \times \prod_{i=1}^{p} k_{i}
$$

where $L_{i}$ is the fixed set of labels for the $i$ th variable in the input space, of a cardinality $\left|L_{i}\right|=k_{i}$; $H_{L_{i} j} \in H, j=1,2$, with $H$ being the set of applicable hedges of a cardinality $|H|=h$; and $S$ is the fixed set of class labels of the output space, of a cardinality $|S|=s$. The modeling task is to select the smallest possible subset of $R_{S e t}$ that characterizes it to a degree as high as possible. This value increases dramatically as input dimensionality increases. This makes it impossible to perform exhaustive search for any moderately sized problem. Even robust but non-exhaustive search techniques such as GAs, may not perform well when $|R|$ is large [19]. This is mainly because many of the rules generated may not cover any data. The subset of interesting rules that at least cover some data may indeed be very small compared with the total; much effort of the search is often wasted in order to find that small subset. However, the search of interesting rules can be considerably reduced if it starts with a rough solution and then a GA is used to optimize this solution.

The question becomes how to find a rough solution efficiently. Fortunately, there already exist approximative rule generation techniques that are, focusing on given data, able to find very accurate rules, without using pure and brute force search. Being data-driven [16], these techniques avoid the empty parts of the input space. There are no restrictions over the fuzzy sets they use, that is, these sets do not have to satisfy any prescribed linguistic interpretation. The resultant approximative rules "point" to places in the search space where desirable descriptive rules potentially exist. These 
approximative rules can then be transformed into descriptive ones with a heuristic method, which is used as the generator of the first population for a GA that will optimize the translation.

Figure 1 shows the basic ideas of the present work. Instead of using a direct descriptive rule induction technique, which is generally rather slow and inaccurate, it is proposed to use a fast and accurate approximative rule induction algorithm first. (The approximative fuzzy model induced can be tuned to improve its modeling accuracy.) The approximative model is then converted into a fuzzy linguistic model that utilizes predefined descriptive sets. It is this conversion process that forms the major work reported herein.

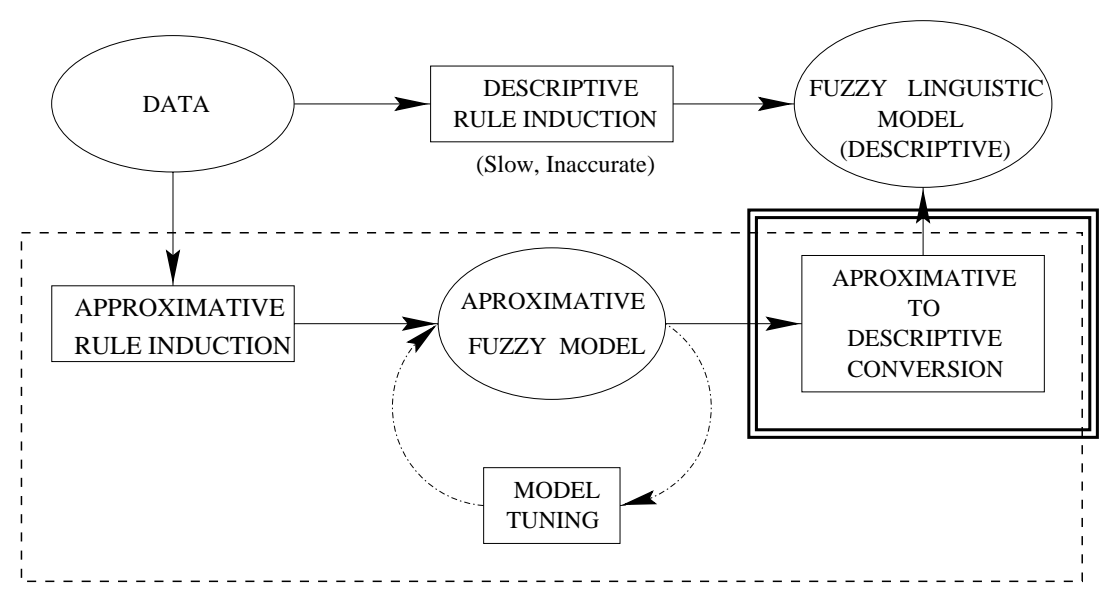

Fig. 1. Descriptive rule generation

Through the use of approximative rules, a vast volume of the search is already done. The GA's effort is directed to perform fine adjustments. The emerging solution, i.e. a descriptive ruleset, can be improved due to the neighbourhood search operators included in the GA or inserted between GA generations. In general, the better the initial translation the less effort the GA will have to apply, as the initial solution is already close to the final ruleset, usually far closer than a random one.

\section{HEDGES}

The application of a linguistic hedge modifies the shape of the membership function of a fuzzy set [20], transforming one fuzzy set into another. The meaning of the transformed set can easily be interpreted from the meaning of the original set and that embedded in the hedge applied.

The definition of hedges has more to do with common sense knowledge in a domain than with mathematical theory. Although a simple linguistic hedge may be used to express different modifications in different applications the general type of the underlying operation remains the same, varying only in terms of detailed parameter settings. For example, concentration/dilation hedges are often implemented by applying a power function to the original set membership values [20], [21]. That is, given the original membership function $\mu_{S}(x)$ of a fuzzy set $S$ and hedge $H$, the membership function of $H \cdot S$ is $\mu_{H \cdot S}(x)=\mu_{S}^{e}(x)$, where the exponent $e$ is greater than 1 for concentration and less than 1 for dilation. Different values can be assigned to the exponent $e$; for hedge EXTREMELY for instance, $e=2$ is used in [20], while $e=8$ is employed in [21].

Conventional definitions of hedges do not result in significant changes on trapezoid fuzzy sets, which are most commonly used for computational simplicity purposes. In particular, the full membership part of a trapezoid membership function does not get changed at all. In this work a different implementation of the hedges is considered, which may be applied to concentrate or dilute an original fuzzy set by shrinking or expanding any parts of the trapezoid. In addition, three new hedges named UPPER, LOWER and MID that do not appear in the literature are also proposed.

A trapezoidal membership function, characterized by 4 parameters $(a, b, c, d)$, consists of three consecutive segments as illustrated in figure 2. The application of concentration/dilation hedges 
should decrease/increase the size of these segments and, therefore, be implemented with the modification being in proportion to the center of the full membership segment $(m)$. The following formalizes these ideas and defines the hedges used in this work.

\section{A. Concentration}

Concentration hedges reduce the size of segments or each part of the membership values of an original set. For a given trapezoidal fuzzy set $S$ with a membership function $\mu_{S}(x)$, the set modified by a concentration hedge $C O N$ should comply with $\forall x \in X, \mu_{C O N \cdot S}(x) \leq \mu(x)$. The parameters of the modified set are therefore defined by

$$
\begin{array}{cc}
m=\frac{b+c}{2} \\
b^{\prime}=m-((m-b) * \beta) & c^{\prime}=m+((c-m) * \beta) \\
a^{\prime}=b^{\prime}-((b-a) * \beta) & d^{\prime}=c^{\prime}+((d-c) * \beta)
\end{array}
$$

where $\beta$ controls the degree of shrinking. In order to reduce the set effectively $\beta$ must satisfy $0<\beta<1$. In particular, the commonly used hedge terms MORE, VERY (see figure 2) and EXTREMELY can be defined as follows:

- MORE reduces the segments to $\frac{2}{3}$ of the original size $\left(\beta=\frac{2}{3}\right)$.

- $V E R Y$ reduces the segments by half $\left(\beta=\frac{1}{2}\right)$.

- EXTREMELY reduces the segments to $\frac{1}{8}$ of the original size $\left(\beta=\frac{1}{8}\right)$.

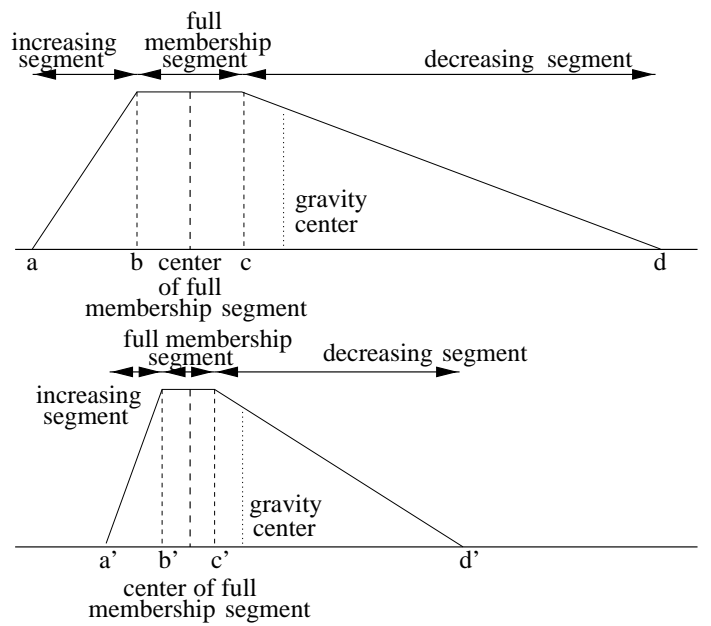

Fig. 2. Parts of a trapezoidal set and application of the hedge VERY

\section{B. Dilation}

Dilation hedges increase the size of segments or each part of the membership values of a fuzzy set. As opposite to a concentration hedge, a dilation hedge $D I L$ should comply with the following intuition: $\forall x \in X, \mu_{D I L \cdot S}(x) \geq \mu_{S}(x)$. The parameters of the modified set are calculated as concentration hedges, but this time the factors will be greater than one:

- GREATLY increases the segments by 2 times the original size $(\beta=2)$.

- LESS increases the segments by $\frac{3}{2}$ of the original size $\left(\beta=\frac{3}{2}\right)$.

Note that the pair MORE and LESS, and the pair VERY and GREATLY are complementary; they cancel each others effect as they express exactly opposite concentration-dilation concepts. No hedge was found in the literature that matches the opposite of EXTREMELY (perhaps REMOTELY could be a candidate for this), but including such a dilation hedge is as simple as setting $\beta=8$. 


\section{Restriction}

Restriction hedges [20], $A B O V E$ and $B E L O W$, are applicable to variables where fuzzy values are ordered. The set modified by applying the $A B O V E$ hedge denotes the set which is "greater than" the original set and that by the $B E L O W$ hedge represents the set which is "less than" the original. The resulting sets are therefore shouldered ones, the left shouldered for BELOW and the right shouldered for $A B O V E$. Their membership functions are defined as follows:

$$
\mu_{A B O V E \cdot S}(x)=\left\{\begin{array}{ll}
x<c & 0 \\
c \leq x<d & 1-\mu_{S}(x) \\
x \geq d & 1
\end{array} \quad \mu_{B E L O W \cdot S}(x)= \begin{cases}x<a & 1 \\
a \leq x<b & 1-\mu_{S}(x) \\
x \geq b & 0\end{cases}\right.
$$

Note that the application of restriction hedges to some fuzzy sets may make no sense. This includes cases where the hedge $A B O V E$ is to be applied to a right shouldered set (or any set whose $\mu(x)=1$ when $x \rightarrow \infty$ ) and similar ones where $B E L O W$ is used to modify a left shouldered set. Such non-sense hedge-set combinations are disallowed, as they always return 0 memberships and hence cause the rules which would otherwise involve them not to fire anyway.

\section{Detailisation}

This proposed new type of hedge splits the original set into three, but keeps the order of these split sets the same as the order of the elements belonging to the full membership segment of the original. These hedges will, therefore, only make sense on variables whose values are ordered, as with the restriction hedges. The resulting three sets $L O W E R \cdot S, M I D \cdot S$ and $U P P E R \cdot S$, arranged in an increasing order, are defined by:

$$
\begin{gathered}
\text { LOWER } S\left\{\begin{array} { l l } 
{ a ^ { \prime } = a } & { b ^ { \prime } = b } \\
{ c ^ { \prime } = b + \frac { b - c } { 3 } } & { d ^ { \prime } = b + \frac { 2 * ( b - c ) } { 3 } }
\end{array} \quad M I D \cdot S \left\{\begin{array}{ll}
a^{\prime}=b & b^{\prime}=b+\frac{b-c}{3} \\
c^{\prime}=b+\frac{2 *(b-c)}{3} & d^{\prime}=c
\end{array}\right.\right. \\
\text { UPPER } \cdot S \begin{cases}a^{\prime}=b+\frac{b-c}{3} & b^{\prime}=b+\frac{2 *(b-c)}{3} \\
c^{\prime}=c & d^{\prime}=d\end{cases}
\end{gathered}
$$

As an example, if the original fuzzy set $S$ is $(0,3,12,14)$ then $L O W E R \cdot S$ is $(0,3,6,9), M I D \cdot S$ is $(3,6,9,12)$ and $U P P E R \cdot S$ is $(6,9,12,14)$.

\section{E. The NOT operator}

Although NOT is not a hedge but a logical operator, in terms of its application effects it may be viewed as a hedge for presentational convenience. This is because the application of this operator to a fuzzy set also changes the shape of the membership function of that set (as $\mu_{N O T \cdot S}=1-\mu_{S}$ ). For this reason, it will be treated similarly as any other hedge hereafter.

Finally, to have an overview of the new hedges introduced above, figure 3 shows the results of applying them to a given irregular trapezoidal fuzzy set.

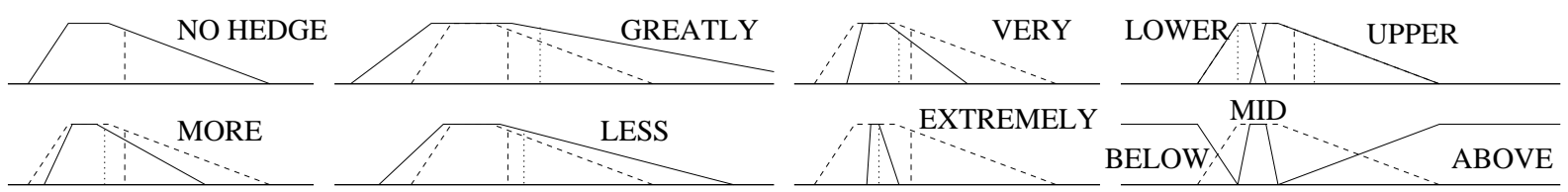

Fig. 3. Hedges applied to an irregular trapezoid and how they change the center of gravity 


\section{Mapping Approximative onto Descriptive Rules}

The main aim of this work is to find an efficient and effective way to translate rules that use approximative sets into rules that use descriptive sets and hedges. The translated rules will be equivalent to the original, or a close equivalent within the limitations of the GA search, with the advantage of having or regaining human-comprehensible interpretation. This it does not matter as to which technique is used to generate the original approximative rules. What is required is a set of approximative rules and the definition of the descriptive fuzzy sets and linguistic hedges. In the experimental studies to be presented later, a hybrid method is used to produce the initial approximative rules. For the use of the proposed heuristic method (for generating the initial descriptive rules) there must also exist a similarity measure between the fuzzy sets used in the approximative rules, which can be of any type, and the descriptive trapezoidal fuzzy sets. Trapezoids are adopted as final descriptive sets for computational efficiency purposes.

To perform the mapping a concatenation of two methods is proposed here. One is based on a heuristic search (As the space of potential descriptive rules can be very large, techniques of branch and bound are applied and so the power of this heuristic method may be rather restrictive.). The other uses a GA to work on the full search space. As evolutionary search usually works better when a good start point has been identified [22], the first method will be employed as the generator of the initial population for the GA, which will then make a finer-grain search. The heuristic translation may not yield spectacular results but it is far better than a random start as will be shown later.

\section{A. Heuristic Approach}

This approach is based on hyperbox intersection with given approximative rules. Descriptive rules, i.e. hyperboxes defined on descriptive sets, are created if they intersect with an approximative rule (or the hyperbox defined by the antecedent approximative sets). This produces a preliminary translation, which is the one often used for explanation purposes in the existing literature [12]. The basic component of this proposed method uses no hedges and serves to introduce the underlying ideas of the heuristic translation. This has been extended to include the use of hedges, but only the basics are explained here with the extensions outlined for presentational simplicity.

This heuristic method works by building a layered graph to represent degrees of intersection between approximative and descriptive sets, using an intersection-based similarity measure. Each layer of the graph consists of a certain number of nodes; each of which represents the amount of intersection between one of the approximative sets of an antecedent variable and one of the descriptive sets of the same variable. Thus, each path of the resulting graph may be interpreted as a possible descriptive rule which coarsely approximates a given approximative one. The amount of similarity between two sets $S_{1}$ and $S_{2}$ is hereafter called the Similarity Value $(I)$ of the two. In this work the similarity used is defined by:

$$
I_{S_{1} S_{2}}=\frac{A\left(S_{1} \cap S_{2}\right)}{\operatorname{Max}\left(A\left(S_{2}\right), A\left(S_{1}\right)\right)}
$$

with $A($ Set $)$ denoting the area of the set Set.

The similarity this value may vary from zero for no intersection to one for equality. When intersection is null the corresponding node is removed from the graph. Incidentally, although the above particular definition is utilized in this work, empirical results have shown that other similarity metrics proposed in the literature [23], [24], [25] may be adopted to take its place without major disruption in the mapping results. However, this definition has proven to be computationally simple and performance-wise robust.

Supported by such a similarity metric, given an approximative rule $Q$ :

$$
\text { IF } x_{1} \text { is } S_{1} \text { AND } x_{2} \text { is } S_{2} \text { AND } \ldots \text { AND } x_{p} \text { is } S_{p} \text { THEN Class }
$$

and a collection of descriptive sets $\left\{L_{i j} \mid j=1,2, \ldots, k_{i}\right\}$ per variable $x_{i}$ the preliminary method to build the graph is summarized in figure 4 . 


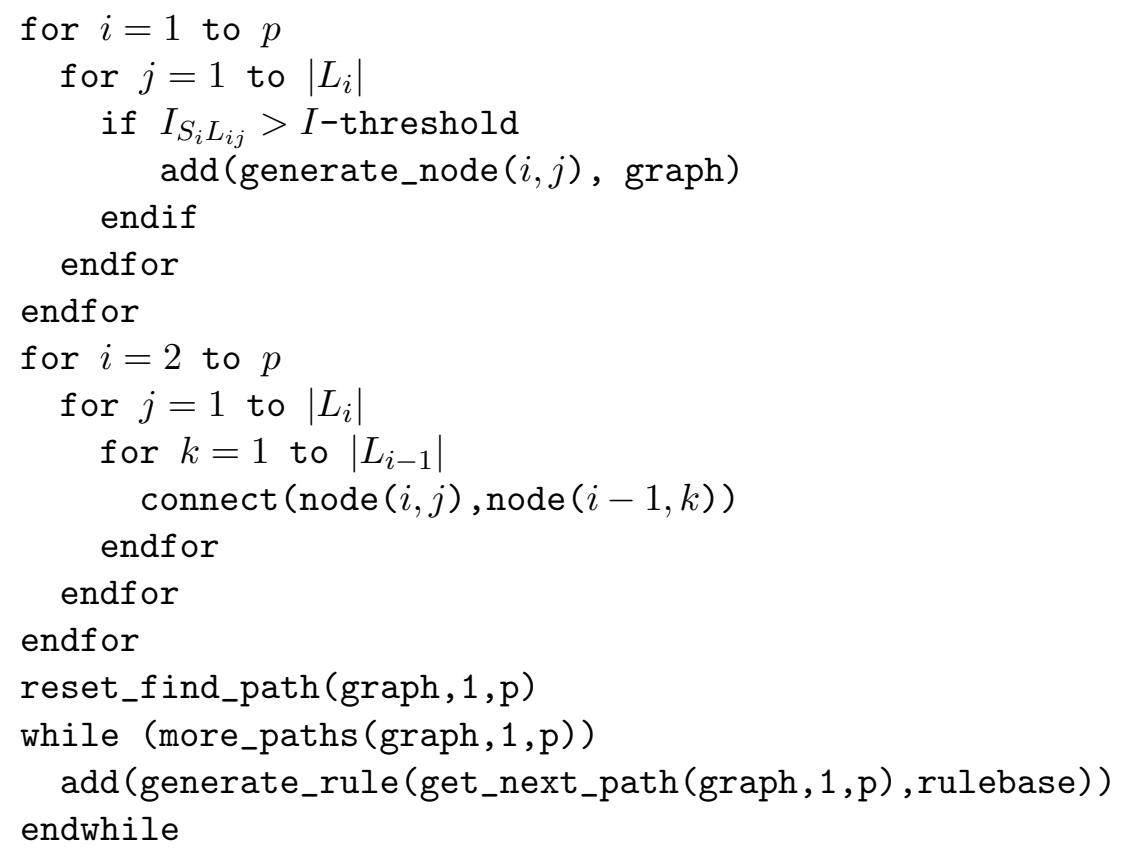

Fig. 4. Graph generation algorithm

To illustrate this basic approach consider the following example. Assume that the input space is two-dimensional. For each of the two input variables, $x_{1}$ and $x_{2}$, three descriptive fuzzy sets are defined such that $x_{1}$ may take a value on either $L_{11}=$ Low, $L_{12}=$ Medium or $L_{13}=$ High, and $x_{2}$ on either $L_{21}=$ Small, $L_{22}=$ Medium or $L_{23}=$ Large. Suppose that the approximative rule to be translated is:

$$
\text { IF } x_{1} \text { IS } S_{1} \text { AND } x_{2} \text { IS } S_{2} \text { THEN } A
$$

where $S_{1}$ and $S_{2}$ are two approximative fuzzy sets defined on the domains of $x_{1}$ and $x_{2}$ respectively and $A$ is a possible class value. The descriptive sets, the grid generated by these sets and the hyperbox covered by the approximate rule are given in figure 5 .

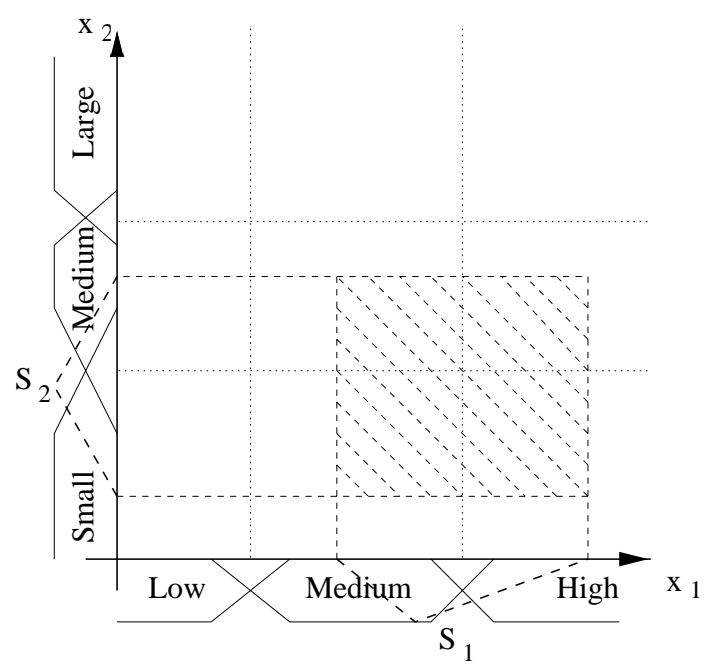

Fig. 5. Approximative rule and descriptive sets

The first layer of nodes is created by taking on the approximative set of the first antecedent of the original rule, in this case $S_{1}$, and then constructing a node for each descriptive set $L_{1 i}, i=1,2,3$ 
(that is, Low, Medium and High) of $x_{1}$ if the similarity measure between $L_{1 i}$ and $S_{1}$ is larger than $I$-threshold (zero by default). Suppose that the measure between $S_{1}$ and $L_{12}$ and that between $S_{1}$ and $L_{13}$ are $I_{S_{1} L_{12}}=0.62$ and $I_{S_{1} L_{13}}=0.43$, respectively. Also, suppose that $S_{1}$ does not intersect $L_{11}$. Thus, two nodes are created, as illustrated under $S_{1}$ in figure 6 .
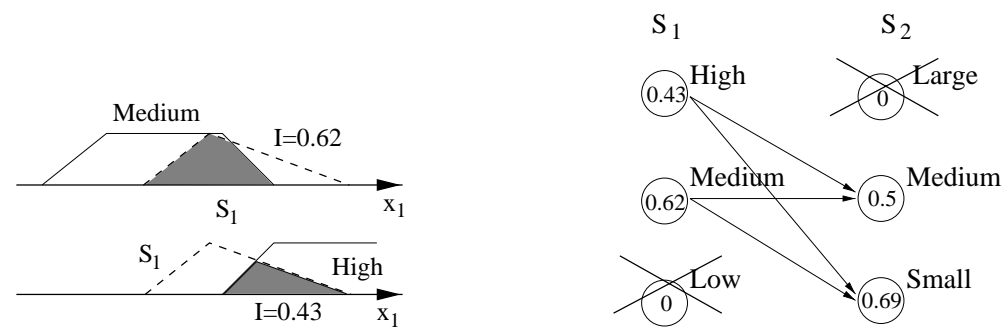

Fig. 6. Intersections of descriptive and approximative sets (left) and graph generation (right)

This process is repeated for each variable that appears in the antecedent of the original approximative rule, resulting in different layers of nodes with each layer corresponding to one variable. Then, all the nodes in one layer are connected to the nodes in the next with the arrow of each link pointing from a previous node to a newly created one, as also shown in figure 6 .

Once the graph is generated paths from any node in the first layer to a node in the last are constructed. Each path becomes an emerging rule, with the antecedent variables taking the labels of the nodes of that path. Thus, the resultant set of descriptive rules which collectively form a preliminary translation of the given approximative rule are:

$$
\begin{aligned}
& R_{1}: \text { IF } x_{1} \text { IS High AND } x_{2} \text { IS Medium THEN } A \\
& R_{2}: \text { IF } x_{1} \text { IS High AND } x_{1} \text { IS Small THEN } A \\
& R_{3}: \text { IF } x_{1} \text { IS Medium AND } x_{1} \text { IS Medium THEN } A \\
& R_{4}: \text { IF } x_{1} \text { IS Medium AND } x_{1} \text { IS Small THEN } A
\end{aligned}
$$

This heuristic method does not ensure a good coverage of an approximative rule unless the threshold used is very low. However, a low threshold potentially implies many nodes and hence many descriptive rules. This implies that the method can be quite sensitive to such parameters settings. Nevertheless, this method is proposed to act as the starting point for the evolutionary search and its accuracy is not of utmost importance. Also, it can be itself improved.

An obvious improvement is to include hedges. In so doing, the number of nodes will, however, increase drastically. This is because the label of a node may now be any combination of a descriptive set and a number of hedges used to modify the set. Even if nodes with a similarity value below the threshold are eliminated, and if the up-ceiling of the number of hedges applicable to a set is limited to two, this may still result in a significant increase of the number of nodes in the graph.

To perform an extra reduction of the graph and hence the number of emerging descriptive rules, various heuristics may be applied to eliminate unwanted nodes. In particular, if some nodes within a layer are similar to each other only one of them would then be needed. Also, external control of the desirable distinctions amongst possible values per input variable, that is the number of nodes permitted per layer, can be used to select those which are most dissimilar between one another. Both methods are implemented in this work; they ensure that the nodes left are different among themselves. Of course, these methods are assisted with the requirement that whatever nodes to be chosen they must attain a high similarity value.

\section{B. GA-based Approach}

While the heuristic approach relies on the use of similarity, the evolutionary computation-based approach proposed here depends on the concept of functional equivalence. It works by searching for a set of descriptive rules that collectively behave like the original approximative rule from which they are translated. That is, for data that is covered by an approximative rule, the found descriptive 
rules will fire with at least the same firing strength as the original. Furthermore, for data that would not cause the original approximative rule to fire, the resultant descriptive rules will either not fire or fire if their consequents comply with the desired output. As indicated before, the search mechanism is herein implemented by a GA.

\section{B.1 Training sets and objective functions}

Each approximative rule may be translated independently. Multiple descriptive rules are considered per approximative rule as, in general, an approximative rule $R$ may not be covered by just one resulting descriptive rule. To implement the translation in this manner, a training subset for each approximative rule $R$ needs to be generated from the original training set (from which the approximative rules were obtained). Such rule training sub-sets are derived via a data selection and enrichment process as introduced below.

Suppose that there are $K_{R}$ emerging descriptive rules that, collectively, form the functional equivalent to a given approximative rule $R$. Given a set $\mathcal{X}$ of the original training examples, for each $x_{i} \in \mathcal{X}$ the firing strength $A F S_{R}\left(x_{i}\right)$ of the approximative rule under translation is calculated. If $A F S_{R}\left(x_{i}\right)>0$ it would be desirable that, if the consequent of this rule is the same as the desired consequent, any of the resulting translated descriptive rules $R_{j}, j=1, . ., K_{R}$ will fire for such an example with a strength $D F S_{R_{j}}\left(x_{i}\right)$ equal to or greater than $A F S_{R}\left(x_{i}\right)$. This kind of example will be hereafter referred to as an example of type one. If, however, $A F S_{R}\left(x_{i}\right)>0$ and the consequent does not match the desired, then the firing strength of each resulting descriptive rule $D F S_{R_{j}}\left(x_{i}\right)$ should be less than, or at worst equal to, $A F S_{R}\left(x_{i}\right)$. This kind of example will be referred to as type two. Furthermore, if $A F S_{R}\left(x_{i}\right)=0$ then, if the example $x_{i}$ is of the same desired consequent as that of the original rule, it is not selected to form the training sub-set (as this example provides no influence in executing this learned rule and is expected to be covered by other approximative rules). If, however, the consequent is different, the firing strength of the resulting descriptive rules should be zero. This last kind of example will be referred to as type three.

Clearly, for any $x_{i} \in \mathcal{X}$ and a given original approximative rule $R, x_{i}$ is selected to form the training subset for translating $R$ if and only if it is an example of either of the three types. Each data point is enriched by the inclusion of its type and its $A F S_{R}\left(x_{i}\right)$. This data selection process allows the GA to enforce the following objectives in performing search for suitable descriptive rules, where $T_{t}, t \in 1,2,3$, denotes the subset of training data of type $t$ :

- $\forall x_{i} \in T_{1} D F S_{R_{j}} \geq A F S_{R}\left(x_{i}\right)$

- $\forall x_{i} \in T_{2} D F S_{R_{j}} \leq A F S_{R}\left(x_{i}\right)$

- $\forall x_{i} \in T_{3} D F S_{R_{j}}=0$

As the classification inference is performed by choosing the output value of the rule that has the highest firing strength, enforcing the above conditions yields a descriptive model that is at least as accurate as the original approximative model. This is because the inequality restrictions allow an increase in the firing strength of the descriptive rules to be learned over correct training data (type one) and a reduction in the firing strength over incorrect training examples (type two). However, in general, not all training examples will satisfy these restrictions and it is the job for the GA to reduce the discrepancies between the descriptive and approximative firing strengths as much as possible.

For efficiency, the GA should be guided to search for a set of emerging descriptive rules of a minimum cardinality. This means that an objective is needed to minimize the number of descriptive rules used to act as the given approximative rule. Also, any difference between the DFS of an emerging rule (within the resulting descriptive rule set) and the AFS of the original approximative rule for each data type should be restricted to be minimum. Hence, another objective is introduced to minimize the variance of individual rule error. This way, the error that may be produced by the 
translated rules will be as much evenly distributed among all rules as possible, thereby avoiding individual rules with a particularly high error.

In summary, in searching for a set of descriptive rules that would jointly function as a given original approximative rule $R$, the GA search will be guided by objectives as listed in table I, where $K_{R}$ is the current number of emerging descriptive rules.

TABLE I

OBJECTIVES

Functional Equivalence Objectives (Minimize)

\begin{tabular}{cc} 
Expression & Description \\
\hline$\sum_{x_{i} \in T_{1}, j=1, \ldots, K_{R}} \max \left(0, A F S_{R}\left(x_{i}\right)-D F S_{R_{j}}\left(x_{i}\right)\right)$ & Error of type 1 training data \\
$\sum_{x_{i}, \ldots, K_{R}} \sum_{x_{i} \in T_{3}, j=1, \ldots, K_{R}} \max \left(0, D F S_{R_{j}}\left(x_{i}\right)-A F S_{R}\left(x_{i}\right)\right)$ & Error of type 2 training data \\
$\left.\sum_{r} \sum_{j=1, \ldots, K_{R}}\left(x_{R}\right)-E_{R_{j}}\right)^{2}$ & Error of type 3 training data \\
$K_{R}$ & Overall error variance \\
\hline & Additional Classification Objectives of rules \\
\hline Maximize Number of Correctly Classified \\
Minimize Number of Incorrectly Classified \\
Minimize Number of Not Covered
\end{tabular}

In this table $E_{R_{j}}$ denotes the individual error of a descriptive rule, and $\delta_{R}$ represents the mean of the individual errors of all the emerging descriptive rules with regard to the original approximative rule $R$. They are defined as follows:

$$
\begin{gathered}
E_{R_{j}}=\sum_{x_{i} \in T_{1}} \max \left(0, A F S_{R}\left(x_{i}\right)-D F S_{R_{j}}\left(x_{i}\right)\right)+\sum_{x_{i} \in T_{2}} \max \left(0, D F S_{R_{j}}\left(x_{i}\right)-A F S_{R}\left(x_{i}\right)\right)+\sum_{x_{i} \in T_{3}} D F S_{R_{j}}\left(x_{i}\right) \\
\delta_{R}=\sum_{j=1, \ldots, K_{R}} \frac{E_{R_{j}}}{K_{R}}
\end{gathered}
$$

There exist in the GA literature several approaches to deal with such problems that have multiple objectives, including the aggregation approach [26], Non-Pareto approach [27] and Pareto-Based approach [26]. The present work adopts the first of these, as it offers a conceptually simpler method by converting multiple objectives into a compounded single objective. In particular the aggregation function used is the Sum of Weighted Global Ratios (SWGR) [28]. The aggregation method first independently normalizes each objective with respect to the best and worst value ever found for it and, then, weights and adds together each objective to form the single overall fitness value.

The individual translation strategy described above has the drawback that, when the individual translations are put together to form the final translated descriptive rule set, the independently translated rules may interfere with each other. Although a close fit of the descriptive rules to the approximative ones may help resolve this problem, this cannot be guaranteed. It is therefore interesting to consider possible alternative translation strategies. 
Instead of translating individual approximative rules one by one, the first possible alternative approach, valid for problems with a limited range of output values (such as classification problems as mainly concerned herein), is to translate one group of all the approximative rules regarding a single output value at a time. In this so-called group approach an AFS value is calculated as the firing strength of the entire subset of rules concerning the same output value, which is defined by the strongest firing strength of all the original approximative rules that characterize the same class. Thus the translation can be done class by class, instead of rule by rule.

For completeness, another version of the GA search strategy is also included here, and termed the global strategy, where all rules for all classes are represented together in each chromosome. That is, a chromosome is itself a whole translation of the given approximative model ${ }^{3}$.

Pure functional equivalence guidance (i.e., the exclusive use of only the objectives of table I) may miss some otherwise possible improvements of the overall performance of the learned ruleset, because of its trying to fit the approximative model rather than to fit the training data. Empirically, for classification problems, it is generally better to use the influence of functional equivalence objectives along with the above classification objectives and to decrease functional equivalence influence as the GA goes on. That is, the search will initially have a strong focus on the improvement of the heuristic translation to get close to the approximative model and later it will concentrate on the satisfaction of the classification-specific objectives. The reason that search is not guided by the classification objectives alone right from the start is to speed up the finding of optimal descriptive ruleset, by first approximating the emerging descriptive rules to a potentially good accuracy level (offered by the good approximative model) and then optimizing them locally.

Finally, it is worth noting that no guarantees may be given to obtain the closest equivalent translation when a GA run terminates. In general, such a guarantee cannot be obtained without performing exhaustive search. However, given limited computational resources, the translations are empirically very close to the original approximative model in function (as shown later).

\section{B.2 Genetic representation and genetic engine}

In this research, the genetic chromosome representation is based on the work as reported in [30]. Basic ideas of this representation and its associated inference mechanism are outlined below; the detailed codification is beyond the scope of this paper and can be found in [31].

The GA adopted here is a steady-state one. The selection of the two parents is done for one by linear ranking and for the other by random choice. Each child replaces a random member of the worst half of the population. The search stops when the best half of the population does not improve for a prescribed number of generations. Diversity is maintained thanks to the random replacement within the worst half of the population.

After a translation process terminates, those possible descriptive rules that did not fire with the training data available are eliminated. Note that there may exist cases where the eliminated rules cover certain training data, but such data is already covered by other rules with higher firing strength, so they did not ever fire. In experimental studies, to be reported next, not all available data is used for training in order to exploit part of the data to check for possible overfitting.

Three different mutation and four different crossover operators have been implemented in order to investigate what combinations may lead to a good translation ${ }^{4}$. The mutation rate and the rate at which a different crossover is used are both allowed to change dynamically. Empirically, the inclusion of this dynamic schema helps improve significantly the performance of the GA employed.

\section{ExPERIMENTAL Results}

This section presents computer simulation results of applying the proposed descriptive techniques to a number of benchmark problems. The experimental background is first described. A simple

${ }^{3}$ This is known as Pittsburgh style GAs [29].

${ }^{4}$ Details of the mutation and crossover operators are omitted here to save the space. 
example in terms of resultant descriptive rules is given next, in comparison to the original approximative rules. Comprehensive results are then reported and analyzed, supported by comparisons with related work.

\section{A. Experimental Background}

To demonstrate the proposed approach at work, benchmark classification problems are used here [32], including the Breast Cancer, Diabetes, New Thyroid, Wine, and Iris datasets. Table II summarizes the set-ups of these datasets.

TABLE II

Classification PROBlems

\begin{tabular}{||l|c|c|c|}
\hline \hline Name & No. of Inputs & No. of Output Classes & No. of Samples \\
\hline Breast Cancer & 9 & 2 & 683 \\
Diabetes & 8 & 2 & 768 \\
Iris & 4 & 3 & 150 \\
New Thyroid & 5 & 3 & 215 \\
Wine & 13 & 3 & 178 \\
\hline \hline
\end{tabular}

A neuro-fuzzy approximative rule induction algorithm ANFIS $^{5}$ [21], which uses bell-shaped approximative sets, and which has been optimized, was trained for these problems. The resulting approximative ruleset is employed as the original set of rules for translation.

As indicated before, the output of the heuristic method is used to act as the generator of the initial population for the GA that performs finer search for the final descriptive rules. To ensure the readability and understandability of the resulting descriptive ruleset, the maximum number of hedges (including the NOT operator) allowed to be applied to a given set is limited to 2 .

For comparison, the pure descriptive induction algorithm as given in [33], which is a form of exhaustive search with different parameter settings is also tested. In particular, this algorithm (referred to as Lozowski's algorithm hereafter) has a parameter that trades off between the model accuracy and the size of learned ruleset. It determines the minimum difference between the firing strengths of any given rules that have the same antecedent but different class values. In the present investigation, this parameter is set up with reference to the number of rules that the heuristic method has generated to ease comparison. Also, for comparison purposes, results obtained by running the standard C4.5 algorithm [34] are included.

To be simple and fair for comparison, for each problem considered the fuzzification was carried out proportionally with respect to the size of the universe of discourse of the individual variables. That is, for each variable, the distance between its maximum and minimum value within the data set is divided such that all of them approximately cover an equal range of the underlying real values, with soft boundaries of course. The fuzzy sets resulting from such a partition are regarded as the given descriptive sets. This is implemented for illustrative purposes and is not necessary in practical applications of the present work. In fact, the whole idea is that the fuzzy partitioning and labelling will be done by user/experts. To demonstrate the effectiveness of this approach the fuzzification scheme used has only 3 labels per variable.

The GA uses a population of 30 rulesets and run for 10000 evaluations (not generations). Note that, to allow comparison, for a given execution the evaluations are divided among the different subproblems or sub-GAs, if applicable. These sub-GAs are GAs running on parts of the translation. For example, for the individual translation strategy a sub-GA is a GA used to translate a particular rule. In group strategy it is a GA running a particular class. The term sub-GA does not apply to the global translation strategy as there is only one problem, i.e. the translation of the whole approximative ruleset. Thus an execution of the global strategy will have 10000 evaluations, while

\footnotetext{
${ }^{5}$ Note that the ANFIS algorithm is considered as one of the best approximative modelers at the moment.
} 
a group execution for 3 classes will have 3333 evaluations per class translation and an execution of the individual translation strategy for 10 rules will have 1000 evaluations for each rule translation.

As GA execution is computationally affordable, it is worthy to execute the genetic search for as many times as possible. This is in order to obtain the best among as large number of different translations as possible to act as the final translation. The figures to be presented below will show the mean error of translated rulesets depending on the number of runs allowed for the GA. Such error measures are obtained using a bootstrapping of 1000 samples over 100 real runs. Experimental results are given for the GA guided by functional equivalence and also for the GA guided by classification (error) rate alone. To avoid possible overfitting each dataset has been separated into a training set containing $75 \%$ of all the given data and a test set comprising the remaining $25 \%$.

\section{B. Example of Transparency Gained by Translation}

To reflect the fundamental differences between approximative and descriptive modeling, an example ANFIS ruleset for the Iris problem [35] and one of its descriptive translations are given here. The approximative rules are:

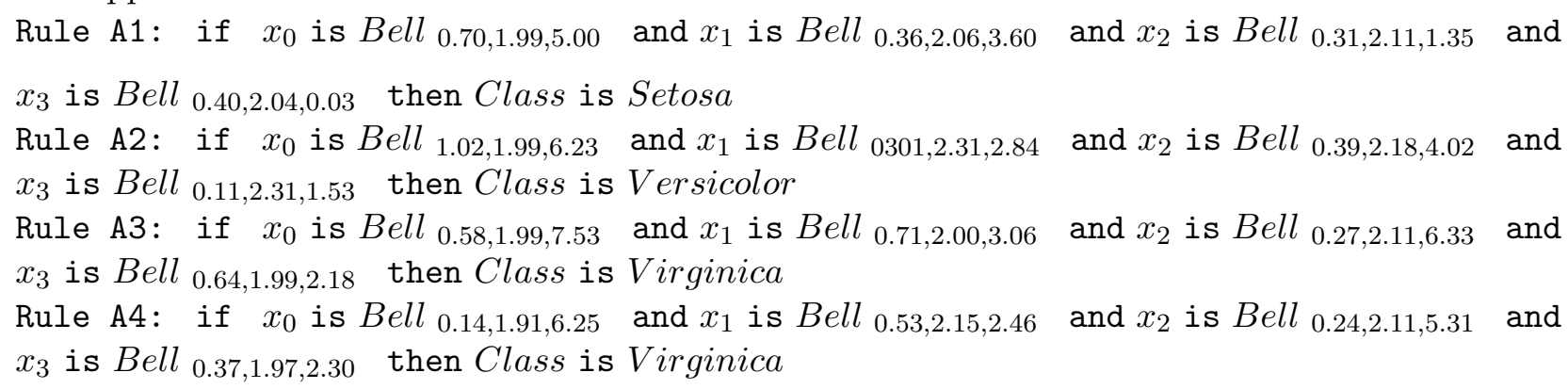

Obviously, these rules are not readable, though they may be generated very rapidly and they may well generalize the given training data.

Suppose that the labels attached to a variable's three possible descriptive sets are named long, medium and short or thin, medium and wide, depending on whether the variable refers to length or width respectively. Each descriptive set may be modified by zero up to two hedges (as defined in section III). Given the approximative rules, the following translated rules may be generated:

Rule D1: IF Petal Width IS Upper Thin THEN Iris-Setosa

Rule D2: IF Sepal Length IS Medium AND Sepal Width IS Greatly Medium AND Petal Length IS Very Medium AND Petal Width IS Very Medium THEN Iris-Versicolor

\section{Rule D3: IF Petal Width IS Lower Greatly Wide THEN Iris-Virginica}

To examine the translation results, figure 7 shows the descriptive partition used in this example. Figures 8 and 9 plot the membership functions involved in the antecedent of the approximative rule A1 and its translation D1 (only the Peal Width has a value, Upper Thin). Also figures 10 and 11 plot rule A2 and its translation D2.

These descriptive rules may appear rather different from the original approximative ones, yet they behave the same functionality. The translated rules are represented in linguistic words with predefined meanings. In using such a rule base, both the interpretation of the inferences performed and the explanation of the fuzzy system itself becomes straightforward. Very interestingly, for this example, the number of resultant descriptive rules is actually less than that of their original, whilst these two rulesets entail the same classification accuracy. Also, two out of the three descriptive rules are more concisely represented than any of the four original rules.

In general, it is very difficult to expect a double translation (which starts from a descriptive model, produces a dataset from this model, generates an approximative model of that dataset and then translates it back to a descriptive model) to reproduce the same original descriptive ruleset 
or even to resemble the original closely. This is more obvious in modeling complex systems where many possible combinations of rules can yield similar rule-firing results. No principled way exists to guarantee that a data driven rule induction mechanism would reproduce exactly the same rules that were first used to create the data. What can be expected most is to be able to generate a set of rules that match the data as closely as possible, hoping that such a set of rules do not differ too much from the underlying one. Although multiple descriptive rulesets may be obtained from one given approximative model, only one optimised is eventually chosen to act as the translation. Thus explanation will be unique for a problem at hand once the translation process is completed.
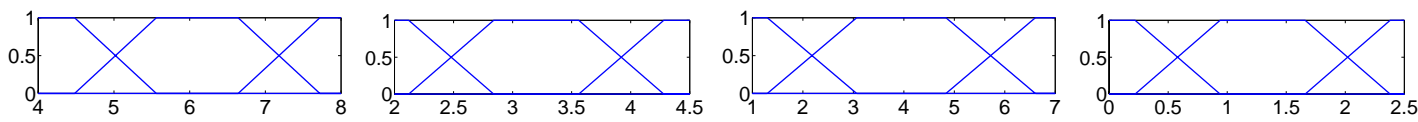

Fig. 7. Descriptive sets for sepal length and width, and petal length and width, respectively (Iris)
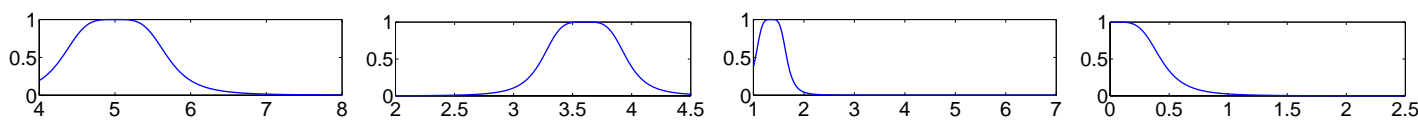

Fig. 8. Fuzzy sets for approximative rule A1 (Iris Setosa)
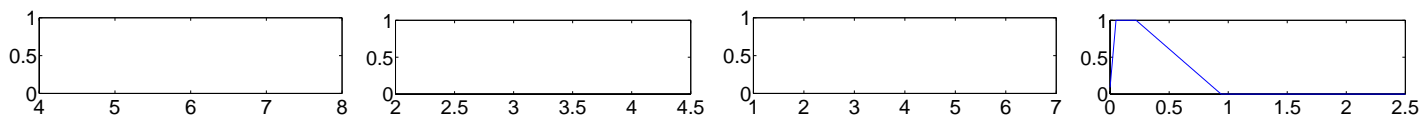

Fig. 9. Hedged sets for descriptive rule D1 (Iris Setosa)
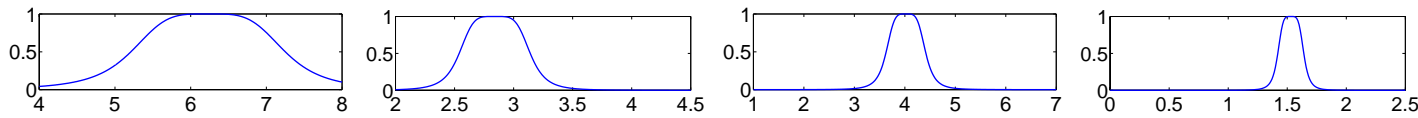

Fig. 10. Fuzzy sets for approximative rule A2 (Iris Versicolor)
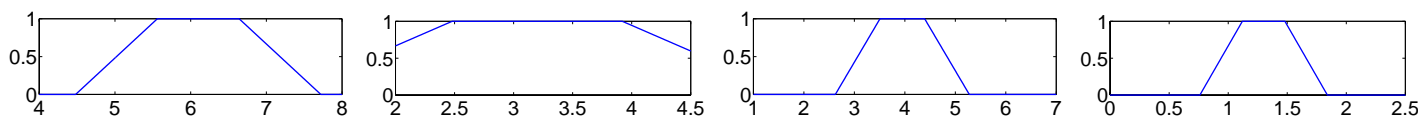

Fig. 11. Hedged sets for descriptive rule D2 (Iris Versicolor)

\section{Results on Model Accuracy}

First of all, it is interesting to investigate the effects of using different translation strategies. Figure 12 collects the results of such experiments on the Diabetes problem, as an example, whilst results on other problems are very similar. It can be seen that the group strategy gave better results than the global and individual ones during the training phase. In testing, all the three strategies performed very similarly overall. However, difference exists in terms of the number of rules needed to achieve the similar test results. Individual translation gave more, sometimes many more, rules as the GA tried to produce good translations locally. The pressure on the search exerted by the number of rules objective on individual strategy was not so high as the pressure by the same objective in carrying out group or global translations, where more data points were involved. When using group 


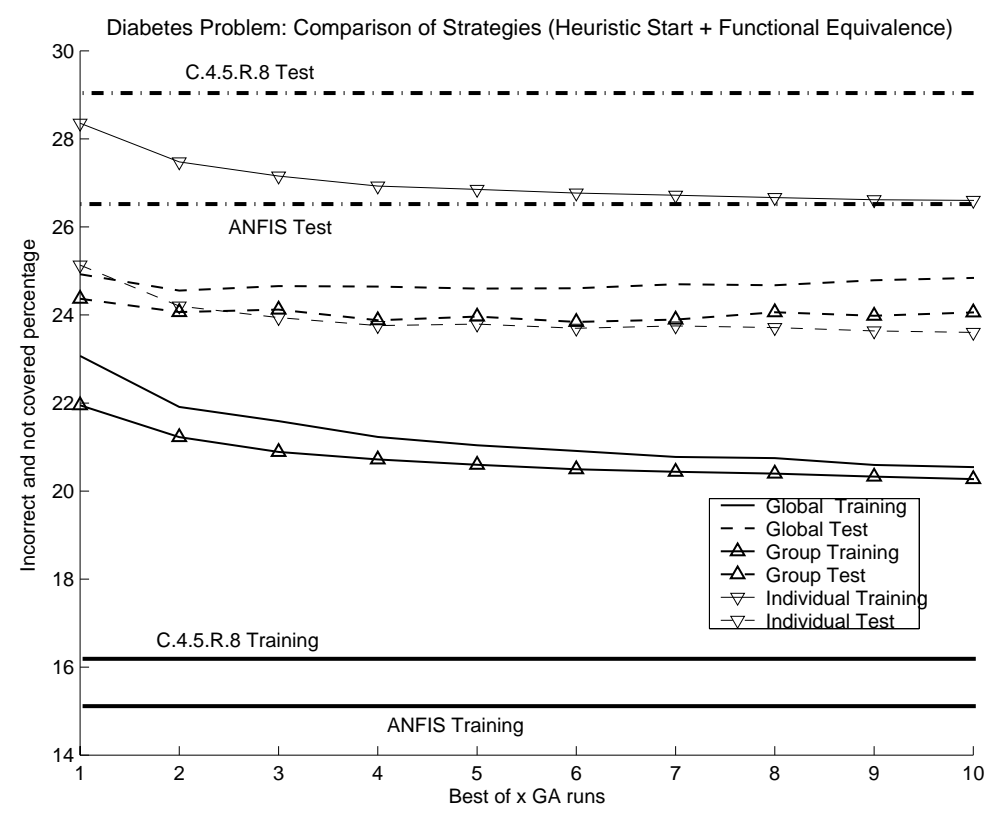

Fig. 12. Classification error vs translation strategy for the Diabetes problem

or global strategies it may happen that several approximative rules can be covered by just one descriptive rule, with potential savings in the number of rules generated.

As group and global strategies allow for more data to be covered, there is more pressure to reduce the number of rules using either of these strategies than the individual strategy. However, this difference in pressure can be compensated to a certain extent as more general strategies have more evaluations to run. The strong point of the individual strategy rests in the fact that it leads to very short and compact rules as can be seen in figure 13 .
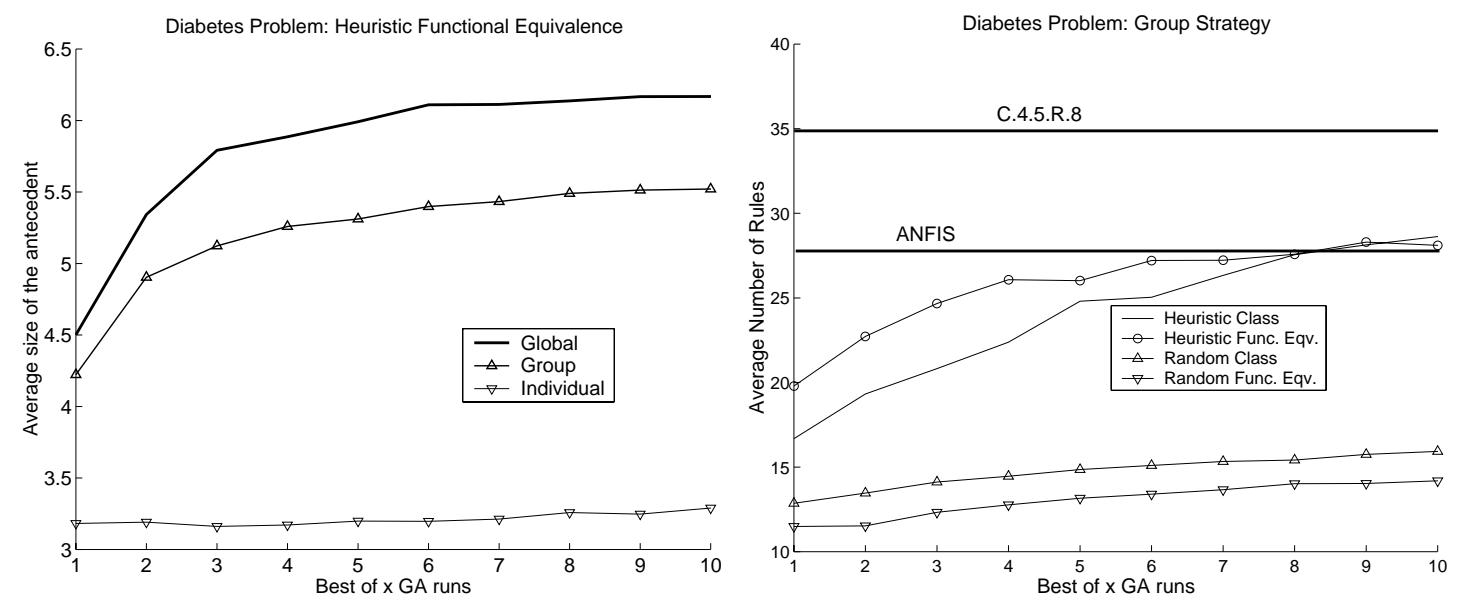

Fig. 13. Mean size of antecedents vs translation strategy (left) and number of rules using the group strategy (right) for the Diabetes problem

The pressure on the number of rules objective can be used to reduce the size of the resultant ruleset. This will make it easier to understand the general behavior of the system under consideration. Nevertheless, in general, a reduced number of rules is likely to result in a reduced classification rate (see figures 13 and 14 or 12 and 15).

As different translation strategies lead to very similar testing performances of the resulting descriptive rulesets, only those results obtained by the use of group strategy are hereafter presented. 


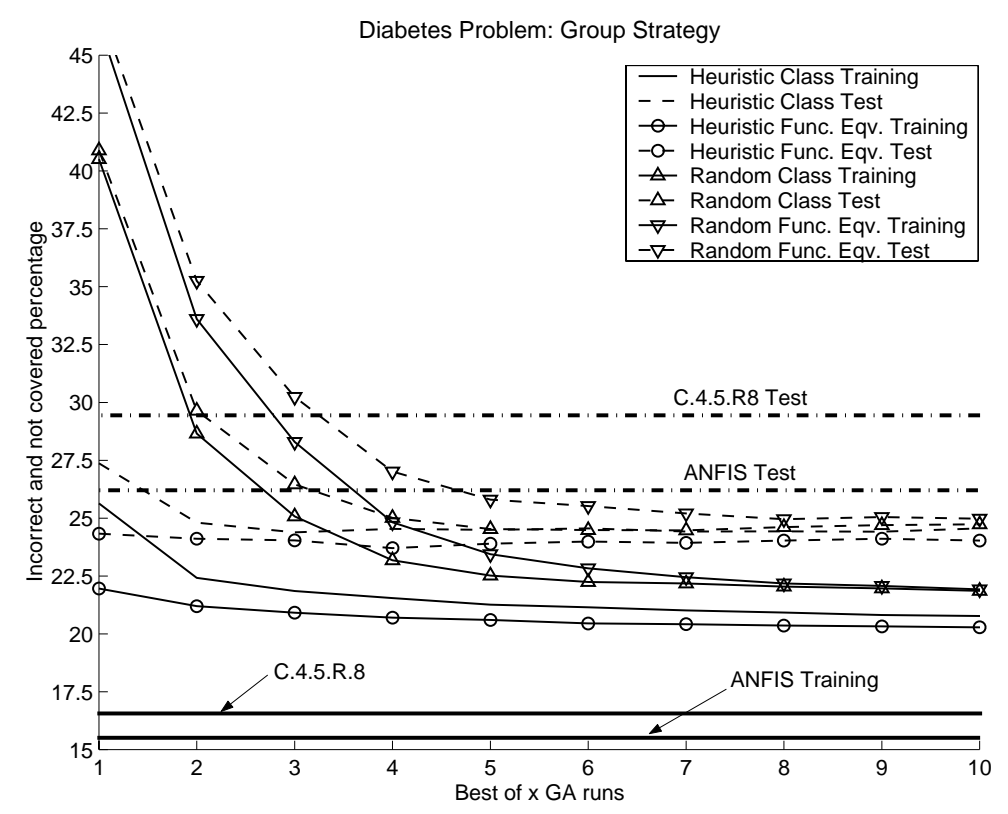

Fig. 14. Classification error for the Diabetes problem using the group strategy

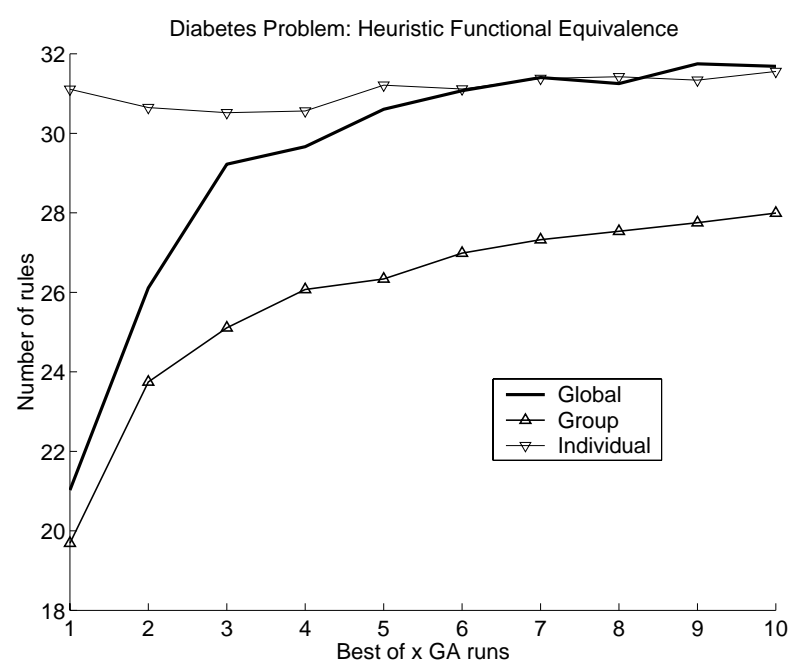

Fig. 15. Number of rules vs translation strategy for the Diabetes problem

Tables III and IV give the results of runs with respect to the following (where Trn, Tst, and Rul respectively stand for the classification error percentage over training data, that over testing data, and the number of rules generated):

a) Starting from a heuristic translation and guiding the GA by classification objectives only.

b) Starting from a heuristic translation and guiding the GA by functional equivalence and classification objectives.

c) Starting from randomly generated rules and guiding the GA by classification objectives only.

d) Starting from randomly generated rules and guiding the GA by functional equivalence and classification objectives.

Within these tables, the most interesting points to compare are those given in the columns concerning items b) and c) above. The former shows the result of what is suggested in this work, and the later shows that of pure data-driven search (no heuristic translation nor approximative model provided). Note that results on the Diabetes problem were collected with two different weights set for the 
number of rules objective, in order to illustrate the impact of this objective upon the translation.

To support the comparison, results of applying a descriptive ruleset which is obtained by the initial heuristic translation alone, and those of using C4.5, ANFIS and Lozowski's algorithm are also provided as given in table $\mathrm{V}$. The work presented in this paper performs well and does so consistently in testing. The accuracy of translated descriptive models is close to that achievable by the optimized ANFIS (comparing table IV and the middle column of table V), and generally outperforms the other descriptive modelling techniques tested (comparing table IV and columns 3 and 4 of table $\mathrm{V}$ ).

Figure 14 shows a graphical comparison, for the Diabetes problem, of the translation results with respect to the number of GA runs. It reveals the difference between different ways of guiding the GA, either starting from a set of descriptive rules obtained by the heuristic translation of the approximative rules or from a set of randomly generated descriptive rules. Graphs plotting the classification errors for the different classification problems appear to be almost the same in their general tendency. They only differ in the actual values of the classification error and in how many GA runs are needed to reach a state where running more GAs will not improve the result. These graphs are therefore omitted here.

TABLE III

MEAN RESUlTS OF TRANSLATIONS FOR ONE GA RUN ONLY

\begin{tabular}{||l||c|c|c||c|c|c||c|c|c||c|c|c||}
\hline \hline \multicolumn{1}{||c||}{ Problem } & \multicolumn{3}{c||}{ Heuristic Class } & \multicolumn{3}{c||}{ Heur Fun. Eqv. } & \multicolumn{3}{c||}{ Random Class } & \multicolumn{3}{c||}{ Rand Fun. Eqv. } \\
& Trn & Tst & Rul & Trn & Tst & Rul & Trn & Tst & Rul & Trn & Tst & Rul \\
\hline Breast Cancer & 2.9 & 7.3 & 9.5 & 1.1 & 5.6 & 8.5 & 14.9 & 18.3 & 9.8 & 10.0 & 14.0 & 9.1 \\
Diabetes (Norm) & 25.0 & 26.8 & 16.9 & 21.9 & 24.5 & 19.8 & 41.0 & 41.5 & 12.8 & 46.2 & 46.8 & 11.5 \\
Diabetes (High) & 28.7 & 29.7 & 8.3 & 25.3 & 27.2 & 9.1 & 48.5 & 48.6 & 9.4 & 46.4 & 46.4 & 3.4 \\
Iris & 1.3 & 4.6 & 5.4 & 1.1 & 4.4 & 5.3 & 5.2 & 9.9 & 5.2 & 3.7 & 6.2 & 5.1 \\
New Thyroid & 12.3 & 13.0 & 7.2 & 7.0 & 7.9 & 6.2 & 54.6 & 55.3 & 7.2 & 45.9 & 46.9 & 6.1 \\
Wine & 3.8 & 10.3 & 10.3 & 1.5 & 5.7 & 10.8 & 45.4 & 47.5 & 10.2 & 36.4 & 39.7 & 10.6 \\
\hline \hline
\end{tabular}

TABLE IV

MeAn Results of the Best translation out of 5 GA RUnS

\begin{tabular}{||l||c|c|c||c|c|c||c|c|c||c|c|c||}
\hline \hline \multicolumn{1}{|c||}{ Problem } & \multicolumn{3}{c||}{ Heuristic Class } & \multicolumn{3}{c||}{ Heur Fun. Eqv. } & \multicolumn{3}{c||}{ Random Class } & \multicolumn{3}{c||}{ Rand Fun. Eqv. } \\
& Trn & Tst & Rul & Trn & Tst & Rul & Trn & Tst & Rul & Trn & Tst & Rul \\
\hline Breast Cancer & 0.8 & 5.7 & 9.3 & 0.7 & 5.6 & 8.3 & 0.8 & 5.7 & 9.1 & 0.7 & 5.7 & 9.0 \\
Diabetes (Norm) & 21.2 & 24.4 & 24.0 & 20.5 & 23.8 & 26.7 & 24.6 & 27.2 & 14.0 & 23.5 & 25.4 & 15.7 \\
Diabetes (High) & 22.7 & 26.0 & 9.8 & 21.4 & 24.2 & 10 & 25.2 & 26.6 & 8.2 & 25.1 & 27.8 & 9.1 \\
Iris & 0.49 & 3.98 & 5.4 & 0.43 & 3.43 & 4.7 & 0.9 & 5.0 & 5.4 & 0.8 & 4.6 & 5.5 \\
New Thyroid & 6.4 & 7.1 & 7.5 & 5.8 & 6.6 & 6.1 & 11.3 & 13.3 & 7.3 & 9.3 & 11.4 & 6.2 \\
Wine & 0.3 & 6.5 & 10.8 & 0.3 & 3.2 & 9.8 & 6.8 & 13.1 & 9.7 & 2.7 & 9.21 & 10.4 \\
\hline \hline
\end{tabular}

This general trend shows that a GA-based translation starting from random rules produces systematically worse results than that starting from the heuristic translation. In addition, the results achievable with a heuristic translation start are far more stable than those obtained with a random start. This supports the need for generating such first crude translation. For simpler problems (e.g., Breast Cancer) both starting points may eventually lead to similar classification accuracy, yet several runs are needed to ensure this similarity. Nevertheless, as shown in table III, if only a single run is executed (say, due to computational resource limit) the results obtained using a randomly generated initial population would be much worse than those obtained using the initial population produced by the heuristic method. It clearly pays off to generate the heuristic translation first and 
TABLE V

RESULTS OF INITIAL TRANSLATION AND OTHER MODELING METHODS

\begin{tabular}{|c|c|c|c|c|c|c|c|c|c|c|c|c|}
\hline \multirow[t]{2}{*}{ Problem } & \multicolumn{3}{|c|}{$\mathrm{C} 4.5$} & \multicolumn{3}{|c|}{ ANFIS } & \multicolumn{3}{|c|}{ Lozowski } & \multicolumn{3}{|c|}{ Heuristic } \\
\hline & $\operatorname{Trn}$ & Tst & Rul & $\operatorname{Trn}$ & Tst & Rul & $\operatorname{Trn}$ & Tst & Rul & $\operatorname{Trn}$ & Tst & Rul \\
\hline Breast Cancer & 1.2 & 7.6 & 29 & $\overline{0.4}$ & $\overline{4.1}$ & 18 & 10.3 & 15.2 & 74 & 25.7 & 23.9 & 94 \\
\hline Diabetes & 16.1 & 29.2 & 35 & 15.7 & 26.6 & 28 & 38.2 & 39.6 & 65 & 33.5 & 32.8 & 43 \\
\hline Iris & 1.8 & 2.6 & 5 & 0.8 & 2.6 & 4 & 4.5 & 10.5 & 11 & 8.9 & 11.6 & 9 \\
\hline New Thyroid & 1.9 & 7.4 & 13 & 3.1 & 1.8 & 4 & 82.6 & 83.3 & 4 & 25.5 & 25.9 & 4 \\
\hline Wine & 0.8 & 2.2 & 17 & 0 & 2.2 & 6 & 39.8 & 44.4 & 21 & 30.1 & 22.2 & 23 \\
\hline
\end{tabular}

to use such a descriptive rule set to act as the generator for the initial population of the GA.

Guidance for the GA by classification error only is also, in general, rather unstable, when compared with the use of functional equivalence guidance. This comparison is shown in figure 16 , where results of 100 runs on classifying the Thyroid problem, with a heuristic start and using the group strategy, are depicted. Following the guidance by classification error alone produces rather poor runs with high error peaks, which actually happens in all tested problems. This unstability of the results can be partially overcome with a higher number of GA runs, though those extra runs are not always affordable computationally. Even if extra GA runs were affordable it would still be better to use the criterion of functional equivalence over all the runs.
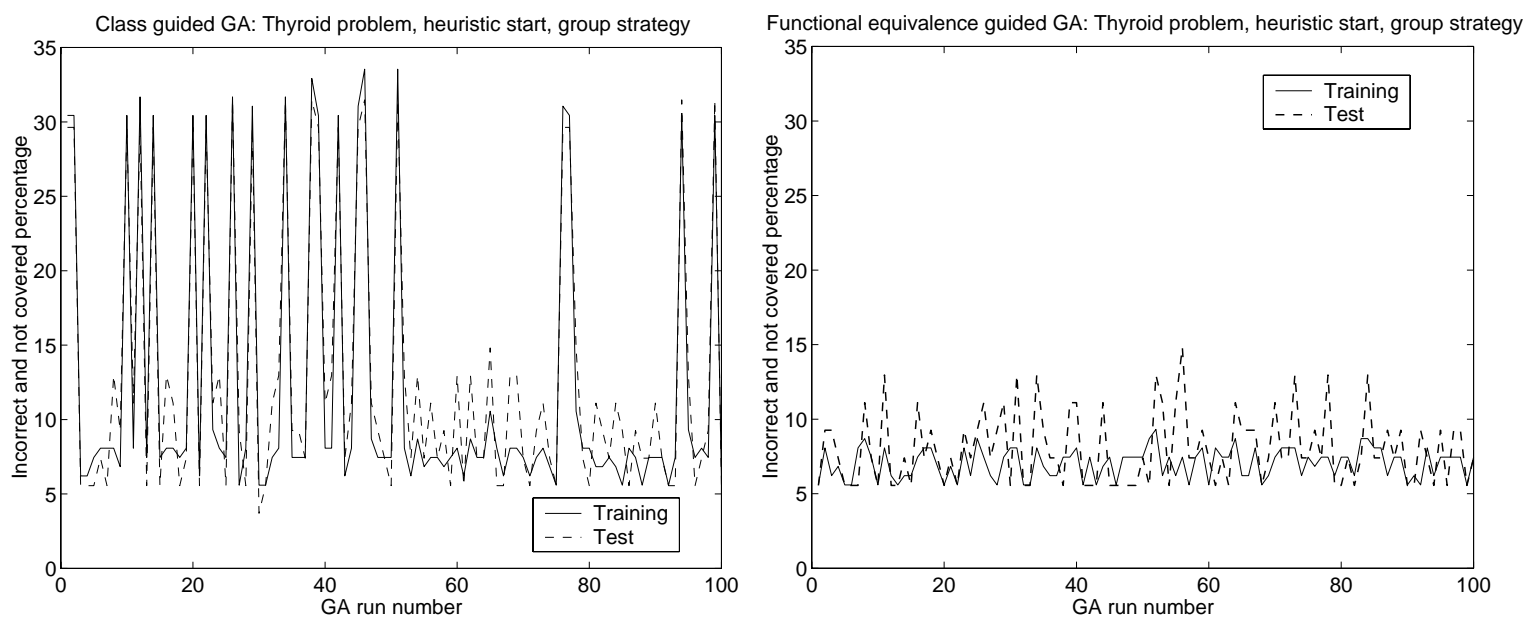

Fig. 16. 100 runs for class guided (left) and functional equivalence guided GA (right) on Thyroid

The above results of a) starting from a heuristic translation and b) making use of functional equivalence reveal an important point. That is, generating an approximative model first (to get required functional equivalence objectives) and then implementing a heuristic translation (to act as the initial population generator for the GA) improves significantly the final descriptive ruleset.

A positive side effect of the way that the rules are codified in the GA is that the size of the antecedent, i.e. the number of conditions in the antecedent, is variable. This implies that there is an implicit attribute reduction going along with the GA-based optimization as shown in figures 13 and 17. This reduction is more evident in high dimensional problems. If desired, the reduction of antecedent conditions may even be explicitly introduced as another optimization objective.

Finally, it is worth noting that, the performance of Lozowski's algorithm, which is an exhaustive search based method, never gets close to that of ANFIS or C4.5. In all the problems tested this algorithm gives poorer results than the approximative modeling methods used and yet requires more rules even for reaching such less desirable results. Compared just to the heuristic translation 


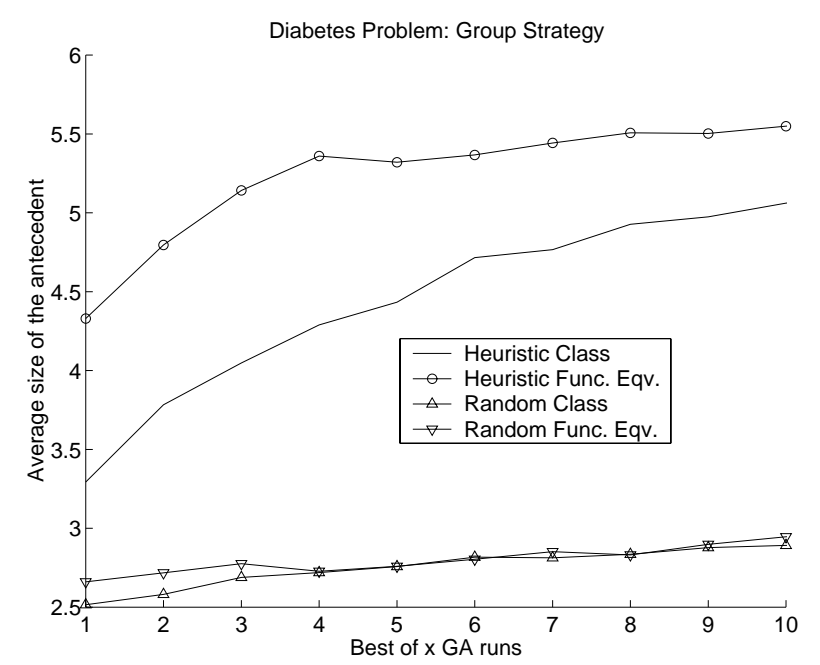

Fig. 17. Mean size of the antecedents for the Diabetes problem using the group strategy

Lozowski's algorithm only defeats it for the Breast Cancer and Iris problems, but its corresponding translated models contain a considerably higher number of rules. However, to be fair with this comparison it must be remembered that Lozowski's algorithm makes use of no hedges.

\section{Conclusions}

A major disadvantage of several existing methods for building descriptive fuzzy systems is that the generation of fuzzy rules is usually made via an exhaustive search throughout the input product space. In addition, the rules produced by pure descriptive methods usually have a low accuracy. However, approximative rule generation methods can be very fast and accurate, through they tend to having difficulties in interpreting the underlying meaning of the data being modeled and in facilitating understanding of the inferences drawn from the resultant models.

In order to generate accurate rules that possess the desirable property of being readily comprehensible to human users and to create such rules in an efficient way, a translation technique from approximative to descriptive rules has been proposed here. The translation is enabled by the use of linguistic hedges to change implicitly the prescribed, meaningful descriptive fuzzy sets, such that the modified will closely resemble the original approximative ones in function. The approximative rules may be themselves created by any standard approximative modeling technique. The modification process is indeed implemented via a functional equivalence guided search. Novel hedges that are useful for supporting such translations are defined in this work. A heuristic algorithm has been presented, which implements a preliminary translation that is employed to act as the generator for the initial population used by a GA to perform the fine grain modifications.

The results obtained so far have demonstrated that the proposed approach does not decrease significantly the accuracy attainable by the original approximative models, and that the descriptive rules obtained are interpretable by humans. It outperforms exhaustive search methods for descriptive rule generation in terms of search efficiency, as searches for descriptive rulesets are herein heuristically guided. In terms of classification accuracy, it also outperforms pure descriptive methods which do not use hedges, whilst it is impractical to run exhaustive search with hedges, as that would complicate even more the usually already huge search space. These results were achieved without important attempts to optimize the GA employed.

\section{ACKNOWLEDGEMENTS}

The first author is partly supported by the Fundación Marín-Blázquez, Spain. Both authors are very grateful to Professor A. F. Gómez Skarmeta of the University of Murcia, Spain, and Professor P. Ross of Napier University, UK, for helpful discussions and assistance in the research reported, 
whilst taking the full responsibility of the views expressed here. Thanks also go to the anonymous referees for their constructive comments which are very useful in revising this paper.

\section{REFERENCES}

[1] S. Abe, "Dynamic cluster generation for a fuzzy classifier with ellipsoidal regions," IEEE Transactions on Systems, Man and Cybernetics, Part B, vol. 28, no. 6, pp. 869-876, December 1998.

[2] L. X. Wang and J. M. Mendel, "Generating fuzzy rules by learning from examples," IEEE Transactions on Systems, Man, and Cybernetics, vol. 22, no. 6, pp. 1414-1427, Nov.-Dec. 1992.

[3] H. Roubos and M. Setnes, "Compact and transparent fuzzy models and classifiers through iterative complexity reduction," IEEE Transactions on Fuzzy Systems, vol. 9, no. 4, pp. 516-524, Aug. 2001.

[4] Lofti A. Zadeh, "The concept of a linguistic variable and its application to approximate reasoning i," Information Sciences, vol. 8, pp. 199-249, 1975.

[5] Rauma T., "Knowledge acquisition with fuzzy modeling," in Proceedings of the 5th IEEE International Conference on Fuzzy Systems, 1996, vol. 3, pp. 1631-1636.

[6] J.-S. R. Jang, Y. C. Lee, and C.-T. Sun, "Functional equivalence between radial basis function networks and fuzzy inference systems," IEEE Transactions on Neural Networks, vol. 4, no. 1, pp. 156-159, January 1993.

[7] D. Nauck and R. Kruse, "NEFCLASS-X- a soft computing tool to build readable fuzzy classifiers," Tech. Rep. 3, BT, July 1998.

[8] M. Setnes and H. Roubos, "Ga-fuzzy modeling and classification: Complexity and performance," IEEE Transactions of Fuzzy Systems, vol. 8, no. 5, pp. 509-522, Oct. 2000.

[9] M. Setnes, R. Babuska, and H. B. Verbruggen, "Transparent fuzzy modelling," International Journal of HumanComputer Studies, vol. 49, no. 2, pp. 159-179, 1998.

[10] M. Setnes, R. Babuska, and H. B. Verbruggen, "Rule-based modeling: Precision and transparency," IEEE Transactions on Systems, Man and Cybernetics - Part c: Applications and Reviews, vol. 28, no. 1, pp. 165-169, Feb. 1998.

[11] J. Valente de Oliveira, "Semantic constrains for membership function optimization," IEEE Transactions on Systems, Man and Cybernetics - Part A: Systems and Humans, vol. 29, no. 1, pp. 128-138, Jan. 1999.

[12] M. Sugeno and T. Yasukawa, "A fuzzy logic based approach to qualitative modeling," IEEE Transactions on Fuzzy Systems, vol. 1, no. 1, pp. 7-31, August 1993.

[13] J. Gómez Marín-Blázquez, Q. Shen, and A. F. Gómez Skármeta, "From approximative to descriptive models," in Proceedings of the 9th IEEE International Conference on Fuzzy Systems, May 2000, pp. 829-834.

[14] J. Gómez Marín-Blázquez and Q. Shen, "Linguistic hedges on trapezoidal fuzzy sets: A revisit," in Proceedings of the 10th IEEE International Conference on Fuzzy Systems, Dec. 2001.

[15] David E. Goldberg, Genetic Algorithms in Search, Optimization 6 Machine Learning, Addison-Wesley", Massachusetts, 1989.

[16] Tom M. Mitchell, Machine Learning, McGraw-Hill, 1997.

[17] H. Ishibuchi, T. Nakashima, and T. Morisawa, "Simple fuzzy rule-based classification systems perform well on commonly used real-world data sets," in NAFIPS '9\%., 1997, pp. 251-256.

[18] S. Dick, A. Kandel, and W.E. Combs, "Comment on "combinatorial rule explosion eliminated by a fuzzy rule configuration' [and reply]," IEEE Transactions on Fuzzy Systems, vol. 7, no. 4, pp. 475-478, Aug. 1999.

[19] H. Ishibuchi, T. Nakashima, and T. Murata, "Performance evaluation on fuzzy classifier systems for multidimensional patter classification problems," IEEE Transactions on Systems, Man and Cybernetics, vol. B, no. 29, pp. 601-608, 1999.

[20] Earl Cox, The Fuzzy Systems Handbook, AP Professional, 1994.

[21] J.-S. R. Jang, C.-T. Sun, and E. Mizutani, Neuro-Fuzzy and Soft Computing, Matlab Curriculum. Prentice Hall, 1997.

[22] P. D. Surry and N. J. Radcliffe, "Inoculation to initialise evolutionary search," in Proceedings of the 3rd AISB workshop on Evolutionary Computation, T. C. Fogarty, Ed. 1996, Springer-Verlag (LNCS).

[23] D. S. Yeung and C. C. Tsang, "A comparative study on similarity-based fuzzy reasoning methods," IEEE Transactions on Systems, Man, and Cybernetics - Part B: Cybernetics, vol. 27, no. 2, pp. 216-227, April 1997.

[24] R. Zwick, E. Carlstein, and D. V. Budescu, "Measures of similarity among fuzzy concepts: A comparative analysis," International Journal of Approximate Reasoning, vol. 1, pp. 221-242, 1987.

[25] Lotfi A. Zadeh, "Similarity relations and fuzzy orderings," in Fuzzy Sets and Applications: Selected Papers by L.A. Zadeh, R. R. Yager, S. Ovchinnikov, R. M. Tong, and H. T. Nguyen, Eds., New York, 1987, pp. 81-104, John Wiley \& Sons, Inc.

[26] Goldberg D. E., Genetic Algorithms in Search, Optimization and Machine Learning, Addison-Wesley, readin Massachussetts, 1989.

[27] J. David Schaffer and John J. Grefenstette, "Multi-objective learning via genetic algorithms," in Proceedings of the 9th International Joint Conference on Artificial Intelligence, Aravind Joshi, Ed., Los Angeles, CA, Aug. 1985, pp. 593-595, Morgan Kaufmann.

[28] P. J. Bentley, Evolutionary Design by Computers, Academic Press Ltd, London, 1999.

[29] S. F. Smith, A learning system based on genetic algorithms, Ph.D. thesis, University of Pittsburgh, 1980. 
[30] D. D. Leich, A New Genetic Algorithm for the Evolution of Fuzzy Systems, Ph.D. thesis, Dept. of Egineering Science, University of Oxford, 1995.

[31] J. Gómez Marín-Blázquez and Q. Shen, "Towards the generation of descriptive fuzzy rules via approximative modelling," in Proceedings of 6th UK Workshop on Fuzzy Systems, 1999, pp. 1-14.

[32] "Uci machine learning databases," Available on web: http://ftp.ics.uci.edu/pub/ machine-learning-databases/.

[33] A. Lozowski, T. J. Cholewo, and J. M. Zurada, "Crisp rule extraction from perceptron network classifiers," in Proceedings of International Conference on Neural Networks, Washington, D.C., 1996, vol. Plenary, Panel and Special Sessions, pp. 94-99.

[34] J. R. Quinlan, C4.5: Programs for machine learning., Morgan Kaufmann, San Mateo, CA, 1993.

[35] R.A. Fisher, "The use of multiple measurements in taxonomic problems," Annual Eugenics, vol. 7, Part II, pp. 179-188, 1936. 